\title{
A Taste of Inequality: Food's Symbolic Value across the Socioeconomic Spectrum
}

\author{
Priya Fielding-Singh
}

\author{
Stanford University
}

Abstract: Scholars commonly account for dietary disparities across socioeconomic status (SES) using structural explanations that highlight differences in individuals' wealth, income, or location. These explanations emphasize food's material value. But food also carries symbolic value. This article shows how food's symbolic value helps drive dietary disparities. In-depth interviews with 160 parents and adolescents and 80 hours of observations with four families demonstrate how a family's socioeconomic position in part shapes the meanings that parents attach to food. These differing meanings contribute to distinct feeding strategies across the socioeconomic spectrum: whereas low-SES parents use food to buffer against deprivation, high-SES parents provision food to fulfill classed values around health and parenting. The findings suggest that an understanding of how families' material circumstances shape food's symbolic value is critical to fully account for dietary differences across SES.

Keywords: food; family; consumption; health; socioeconomic status; qualitative methods

Citation: Fielding-Singh, Priya. 2017. "A Taste of Inequality: Food's Symbolic Value across the Socioeconomic Spectrum." Sociological Science 4: 424-448.

Received: June 15, 2017

Accepted: July 2, 2017

Published: August 10, 2017

Editor(s): Mario Small

D0I: 10.15195/v4.a17

Copyright: (C) 2017 The Author(s). This open-access article has been published under a Creative Commons Attribution License, which allows unrestricted use, distribution and reproduction, in any form, as long as the original author and source have been credited. (c)(i)
TEQUALITY manifests in what we eat. Research shows that significant diet dis1 parities in the United States follow a socioeconomic gradient (Turrell et al. 2002; Kanjilal et al. 2006; Hanson and Chen 2007; Wang et al. 2014). Scholars commonly trace these disparities to either individual economic attributes that impact individuals' abilities to afford a healthy diet (Darmon and Drewnowski 2008) or to ecological factors that delimit the accessibility of healthy foods (Caspi et al. 2012). Both explanations rest on the assumption that food's value is solely material.

But food also has significant symbolic value (Bourdieu 1984; DeVault 1991; Cairns and Johnston 2015; DeSoucey 2016; Beagan et al. 2017). A rich scholarship on socioeconomic status (SES) and consumption highlights the ways in which food provides more than mere physical sustenance: what people eat serves critical social, cultural, and emotional functions (Johnston and Baumann 2010; Johnston, Szabo, and Rodney 2011). Fully understanding socioeconomic differences in people's food choices therefore requires answering two related questions: First, how do individuals across the socioeconomic spectrum assign symbolic value to food? Second, how does that symbolic value shape their dietary choices?

I answer these questions using in-depth interviews with 160 parents and adolescents as well as 80 hours of observations with four families across the socioeconomic spectrum. I demonstrate how parents' food provisioning practices are in part guided by the symbolic value food provides them and how that symbolic value is shaped by families' material conditions. "Symbolic value" refers to the symbolic meanings attached to food (May 2011). For low-SES parents, food serves as a symbolic antidote to a context of deprivation. Daily life strips low-SES parents of opportunities 
to meet adolescents' myriad material desires (Pugh 2009). Food can be an important exception: low-SES parents can often oblige adolescents' inexpensive food requests. As a result, low-SES parents use food to compensate for other domains of scarcity, thereby emotionally satisfying adolescents and bolstering parents' own sense of worth as responsible caregivers. Yet meeting these priorities can undermine parents' professed dietary aspirations for their adolescents.

Among high-SES families, food takes on a different-but equally potentsymbolic value. Whereas low-SES parents derive feelings of competence by fulfilling adolescents' dietary desires, high-SES parents find a sense of worth as caregivers in curtailing adolescents' dietary wishes that parents deem unhealthy. For high-SES parents, who raise their adolescents in contexts of abundance, food offers an ongoing medium for teaching restraint and delayed gratification. High-SES parents therefore regularly deny adolescents' requests for particular foods and drinks, cultivating adolescents' palettes for the "right" foods and signaling to themselves and to others that they are transmitting the "right" values.

For middle-SES parents, who find themselves in a liminal context of neither abundance nor scarcity, food's symbolic value vacillates between its meanings at the two ends of the socioeconomic pole. Middle-SES parents aspire to use food like high-SES parents in an effort to transmit similarly classed values; however, these parents' more limited circumstances mean that they, at times, also use food to compensate for particular types of deprivation. Middle-SES parents' use of food underscores how food's symbolic meaning relates to the material conditions within which parents raise their children.

This understanding of food's symbolic value shows that socioeconomic differences in diet do not result from high-, middle- and low-SES parents' differing valuations of their adolescents' diets or health (Wright, Maher, and Tanner 2015). Most parents across the socioeconomic spectrum hold dietary goals for their adolescents. However, families' material conditions, in addition to positioning them within contexts that impact their abilities to access and afford food for their families, help shape the different meanings that parents attach to food. These differing meanings not only reinforce discourses that highlight parents' personal responsibility-and their failings-for their adolescents' diets, but they also simultaneously obscure the structural inequalities at the root of dietary disparities.

\section{Background}

\section{Socioeconomic Status, Families, and Diet}

The assertion that socioeconomic status is a "fundamental cause" of diet-related health disparities is supported by a body of epidemiological research showing that diet quality and diet-related health disparities among adults and adolescents in the United States follow a socioeconomic gradient (Lutfey and Freese 2005; Darmon and Drewnowski 2008; Phelan, Link, and Tehranifar 2010; Kant and Graubard 2013; Wang et al. 2014). Scholarship examining the origins of these disparities highlights important structural determinants of diet, including differential access to healthy 
food (Caspi et al. 2012; Evans et al. 2015) and the unaffordability of healthy food (Darmon and Drewnowski 2008).

These structural determinants contribute to parents' food provisioning by impacting the means available to parents and the strategies they can employ to accomplish this central caregiving task (Brannen and Nilsen 2005; Gillies 2005; Wills et al. 2011; Daniel 2016). Foundational to a growing body of scholarship on families and food is the finding that parents and adolescents use food for more than physical nourishment. Feeding and eating bestows parents with important emotional benefits (Cairns, Johnston, and MacKendrick 2013), signals status and distinction to others (Johnston and Baumann 2010), allows parents to communicate meaning and care (Gillies 2007), and helps caregivers derive a sense of worth (Tubbs, Roy, and Burton 2005; Lindsay and Maher 2013).

In adolescence, a life stage characterized by increased preferences for and autonomous access to less healthy food choices (Sargent et al. 2002), food is similarly symbolically important. Food offers adolescents a medium for identity formation (Miles, Cliff, and Burr 1998), status signaling (Johnston, Rodney, and Szabo 2012), and in-group belonging (Wills et al. 2009). Adolescents' food preferences and practices are shaped not only by food habits within families but also by external influences, including targeted marketing by the food industry (Gantz et al. 2007; Leibowitz et al. 2012) and peer norms (Stead et al. 2011). With these forces helping to cultivate adolescents' preference for calorie-dense fast foods, parents face new challenges related to their adolescents' diets (Croll, Neumark-Sztainer, and Story 2001).

Research suggests that socioeconomic status underpins how parents navigate these dietary changes associated with adolescence (Eldridge and Murcott 2000; Kaufman and Karpati 2007). Studies have shown variation in the amount of responsibility for and level of control parents report exercising over teenagers' food consumption. Backett-Milburn et al. (2010) find that whereas low-SES mothers largely view teenagers' food practices as progressively their teenagers' own responsibility and their preferences for junk foods as beyond parental control, middle-SES parents report molding teenagers' food practices, tastes, and manners "in the right direction" as parents' responsibilities. This research underscores how what is consumed within families is not only a product of negotiations between parents and adolescents but that such negotiations simultaneously reflect the socioeconomic conditions within which they take place. The current study advances this line of work by examining how socioeconomic conditions shape the meanings that parents attach to adolescents' food requests.

\section{Parenting, Feeding, and Health}

Although food provisioning is a key component of caregiving across the socioeconomic spectrum, parents' food provisioning strategies are in part shaped by the increasingly widespread discourses that place the bulk of responsibility on parents to feed their adolescents in ways that ensure their current and future health (Brenton 2011; Kinser 2017). These normative expectations are part and parcel of an increasingly prevalent intensive mothering ideology, or the widespread expectation 
that mothers should spend extensive time and energy cultivating children and tending to their needs (Hayes 1998). As the stakes around feeding have been raised, mothers have come to be largely viewed as personally responsible for feeding their children "healthy" food and protecting them against "unhealthy" influences (Cook 2009; Zivkovic et al. 2010; Cairns et al. 2013). Research shows that many mothers feel that they fall short of such ideals, with some even seeing the flaws inherent in these discourses. At the same time, many mothers still feel accountable to the normative demands of intensive motherhood, particularly as they relate to food provisioning (Romagnoli and Wall 2012).

Amidst these prevailing discourses and exceedingly high expectations for parenting, feeding, and eating, I ask: how do parents of differing socioeconomic circumstances approach their adolescents' diets? Building on prior research demonstrating the central role of food for parents of differing socioeconomic circumstances (DeVault 1991; Lindsay and Maher 2013; Bowen, Elliott, and Brenton 2014; Cairns and Johnston 2015), I show how food's symbolic value to parents is in part derived from their material circumstances and contributes to their provisioning strategies and adolescents' consumption.

\section{Data and Methods}

\section{Data Collection}

This study draws on qualitative data that I collected in two research phases in the San Francisco Bay Area. Phase one involved 160 interviews within 74 families with adolescents across socioeconomic status (24 low-SES, 24 middle-SES, and 25 high-SES families). I conducted all interviews between January 2015 and May 2016. Inclusion criteria for families included having a child between the ages of 12 and 19 who lived at home. I categorized families by SES using a composite measure of education and income (Cooper 2014). ${ }^{1}$ Families were high-SES if at least one parent had a college education and family income was above 350 percent of the California poverty line. In most middle-SES families, both parents had at least a high school education and household income was between 180 to 350 percent of the poverty line. Most middle-SES parents had some form of additional education, typically a vocational or associate's degree. All low-SES families were below 180 percent of the poverty line and neither parent had more than a high school degree. Most low-SES families received public assistance. Table 1 shows the sociodemographic composition of the full interview sample.

Within each socioeconomic group, I included comparable numbers of families from different ethnoracial backgrounds (white, black, Hispanic, Asian), parental composition (single-parent and two-parent households), occupational status (dualand single-income households), and immigrant status (native- and foreign-born parents). ${ }^{2}$ Incorporating this intrasocioeconomic variation enabled an exploration of how these characteristics interacted with socioeconomic status to shape parents' food provisioning.

I recruited families primarily using purposive sampling (Small 2009). At the time of recruitment, families were told that they would be participating in a study about 
Table 1: Sociodemographic composition of interviewed families.

\begin{tabular}{lccccc}
\hline Socioeconomic Status & White & Hispanic & Black & Asian & Total \\
\hline High & 8 & 7 & 5 & 5 & 25 \\
Middle & 8 & 7 & 5 & 5 & 25 \\
Low & 5 & 8 & 6 & 5 & 24 \\
Total sample & 21 & 22 & 16 & 15 & 74 \\
\hline
\end{tabular}

families and food that involved interviews with parents and adolescents. Although self-selection into the study is an inherent limitation of this project, I sought to ensure diversity among participants through targeting different channels of recruitment and by recruiting through both parents and adolescents (Jiménez 2017). I recruited one-third of families through a public high school (Hillview Central) where teachers, coaches, administrators, and parents connected me to parents and adolescents. ${ }^{3}$ I recruited the other two-thirds of the sample throughout the Bay Area. I approached low-SES parents at food banks, toy drives, and shopping outlets. I identified middleand high-SES parents and adolescents through professional email lists, flyers, and personal contacts. I used snowball sampling, capping referrals at two in order to stretch the socioeconomic and ethnoracial range of the sample. I paid families $\$ 60$ in cash for participating.

Within each family, I conducted separate, private one to two hour interviews with at least one parent and one adolescent to examine how both parents and adolescents independently and interactively shaped family diet. ${ }^{4}$ I developed, tested, and refined the interview guides in November and December 2014 before beginning formal data collection in January 2015. Interviews with parents were semistructured, with questions about grocery shopping, meal patterns, food provisioning priorities and challenges, and their adolescent's role in shaping family diet. I always interviewed the primary caregiver, which for most families was the mother $(N=$ $69)$, though I also interviewed fathers $(N=15)$. Interviews with adolescents $(N=$ 76) involved questions identical to parent interviews in addition to adolescents' perceptions of family diet and independent food choices. The online supplement contains parent and adolescent interview protocols.

I sought to lessen the influence of social desirability on parents' and adolescents' responses by conducting interviews in private and using open-ended questions that allowed respondents to guide the conversation and discuss topics most salient to them. I framed the study as being about "food" rather than about "health" and did not mention terms such as "healthy" or "unhealthy" during the interview before respondents brought these terms up themselves. In addition, interviewing multiple members of each family allowed me to triangulate and verify data offered by each respondent. All interviews were transcribed verbatim and anonymized. I took field notes during all interviews and wrote interview summaries to enable reference to the entire sample during analysis.

Phase two of data collection involved ethnographic observations of 4 families (2 low-SES, 1 middle-SES, and 1 high-SES) with whom I had previously conducted interviews. I selected families based on their socioeconomic background and differ- 
ing food provisioning strategies. I conducted 15 to 22 hours of observations with each family over two months. To address social desirability, I informed families at the outset of observations (with institutional review board approval) that I was interested in observing various aspects of family life while not emphasizing food. Families were thus less specifically attuned to observations of their food practices. Following their participation in the study, I explained to them that food had been a primary focus. No families complained upon learning this; to the contrary, all four echoed the centrality of food to family life.

Observations typically lasted three to four hours at a time, and writing field notes required 8 to 10 hours for each period of observation. I observed families at home as well as during diverse outings, accompanying them on trips to supermarkets, birthday parties, church services, nail salons, and back-to-school nights. In addition to observing mealtimes, my observations also captured less formal eating occasions, such as quick trips to the drive-through or breakfasts on the go. Importantly, spending multiple hours with families allowed me to collect data on family members' exchanges around food, including discussions, arguments, and negotiations. I observed parents interacting with each other and with adolescents as well as adolescents engaging with their siblings and peers. Consistent with the experiences of other family ethnographers, the more hours I observed, the less my presence seemed to influence family members' behaviors (Lareau 2003; Cooper 2014). Families fought, argued, played, gossiped, and went about their daily lives with little regard for my presence. I paid each family $\$ 300$ in cash at the end of their participation.

\section{Data Analysis}

I analyzed interview and observational data using qualitative content analysis, an inductive, dynamic form of analysis that is oriented toward understanding both the manifest and latent content of interview data (Graneheim and Lundman 2004). Overall, I engaged in five rounds of coding, the first following the completion of the interviews and the next four rounds of coding following the completion of the family observations.

First, an initial open coding of the interview data suggested similarity in how parents valued adolescents' diets and dietary health but variation in how they provisioned food. I therefore developed a coding scheme that captured parents' aspirations for their adolescents' diets as well as food-related challenges that parents faced with their adolescent, including whether their adolescent requested "unhealthy" foods and how parents responded to these requests. This second round of focused coding (Charmaz 2006) revealed that adolescents across families requested foods that parents did not want them to consume; however, parents differed in how they responded to such requests. These different approaches were patterned by parents' SES, with most high-SES and some middle-SES parents declining requests and most low-SES parents obliging them. Through rereading and recoding the transcripts, I analyzed if such requests related to differences in parents' accounts of food access, affordability, or nutritional beliefs. These factors did not adequately explain the different approaches, but this round of coding facilitated the generation of a 
hypothesis that what food meant to parents in part shaped their responses to their adolescents' food requests. In a fourth round of coding, I identified passages where parents discussed what food meant to them and how they used food, particularly with their adolescents. I used the coded passages to generate different subcategories of symbolic value. I then conducted a fifth round of focused coding to sort all passages that captured these subcategories. Next, I collapsed these coding categories into two primary forms of symbolic value: "compensatory" and "disciplinary." I mapped the different values and corresponding provisioning approaches onto familial characteristics to ascertain along which dimensions food's symbolic value varied, engaging with existing theory and empirical research-primarily within the fields of the sociology of consumption, family, and health-to contextualize this variation.

The major axis of differentiation for food's symbolic value was socioeconomic status. However, as expected, parents' symbolic ascriptions to food did not always perfectly correlate with their SES. Whereas high- and low-SES families' approaches largely diverged, the heterogeneity of the middle-SES sample meant that food's symbolic value shifted between and even within these families. I highlight these shifting meanings as well as inter- and intrafamily nuances in the Findings section on middle-SES families. Another important point of variation was between mothers and fathers, consistent with scholarship underscoring the importance of food work to motherhood and a core part of maternal labor and identity (DeVault 1991; Cairns et al. 2013). I attend to this varation in the Findings section examining high-SES families. That differences across race or ethnicity did not emerge supports the explanation that it is families' material circumstances, rather than ethnic or cultural origins, that primarily drive food's varying symbolic meanings to parents.

I analyzed the 160 parent and adolescent interviews to descriptively map out and categorize food's symbolic value across my sample. However, given this article's focus on food's symbolic value to parents, I draw exclusively from the 84 parent interviews and 80 hours of family observations in the Findings section. Adolescent interviews were critical to understanding the nature of adolescents' requests and parents' strategies, but parents' reports elucidated the meanings that caregivers attached to food. Finally, it is important to note that parents and adolescents in this study freely used the terms "healthy" and "unhealthy" when discussing food. This is consistent with other studies of family diet (Chapman and Beagan 2003; Beagan et al. 2017). Rather than assuming or imposing my own definitions of these and related concepts on the data, I used interviews and observations to let respondents discuss and define these concepts in their own terms. Thus, the use of such terms in the Findings section reflects parents' perspectives and understandings rather than offering an objective measure of healthy or unhealthy food. 


\section{Findings}

\section{Parents' Food Provisioning Ideals across the Socioeconomic Spectrum}

Across the socioeconomic spectrum, most parents cared about their adolescents' diets and dietary health. Many parents mentioned both the general importance of healthy eating as well as efforts to ensure their adolescent consumed a healthy diet. This highlights the increasing prevalence of discourses about diet and health in addition to parents' internalized feelings of responsibility for their adolescents' diets. Most interview respondents freely and casually used the terms "healthy" and "unhealthy" throughout interviews. When asked at the end of the interview what they meant by these terms, almost all parents—regardless of SES, race, or gendertalked about healthy and unhealthy eating in a similar manner, revealing the ascendancy of what can be considered a "mainstream" or dominant healthy eating discourse (Ristovski-Slijepceniv, Chapman, and Beagan 2008; Beagan et al. 2017). Vegetables, fruits, whole grains, fish, and lean meats were commonly described as "healthy," and some parents' definitions also encapsulated the importance of fresh, homemade, or whole foods. In contrast, unhealthy eating was generally agreed to involve "junk foods," including soda, candy, chips, and fast food, as well as foods high in fat, salt or sugar.

Shared understandings of eating healthy were accompanied by parents' shared beliefs that adolescents should eat a healthy diet: 90 percent of high-SES parents, 85 percent of middle-SES parents, and 70 percent of low-SES parents noted the importance of a healthy diet for their adolescent's current and/or future health. As Skylar Gonzales Sullivan, a high-SES Hispanic mother, explained, "food is an investment in our long-term overall health." Similarly, Miranda Kirk, a low-SES white mother, emphasized the link between diet and health:

You are what you eat. Food is your medicine. As a mom, you tell them as much as you possibly can throughout their life. Your health is gonna depend on what you put into your body. Period.

Despite these similar beliefs, parents differed across the socioeconomic spectrum in their provisioning approaches. Public health scholarship suggests that these SES-based differences should be largely (if not entirely) explained by differences in geographic and financial access to healthy foods (Drewnowski and Specter 2004; Caspi et al. 2012). Yet within this sample, neither access nor affordability could fully account for the differences between families. Most of the families in my sample did not reside in food deserts. ${ }^{5}$ Families reported similar food access: 72 out of 74 families lived within two miles of a supermarket, and all but two families had a car that they used regularly. The remaining two families lived within half a mile of a grocery store. Moreover, all of the parents in my sample reported having access to an affordable supermarket nearby. Of the myriad challenges and constraints that parents reported relating to their adolescents' diet, food access was not one of them. Thus, although food access is undoubtedly an issue for other populations (Gordon et al. 2011), it cannot fully account for differences within this sample. 
Affordability offers a more promising explanation of dietary differences. Finances emerged as a meaningful topic in interviews with parents across the socioeconomic spectrum. Although high- and middle-SES parents reported being cost conscious, most stated that food quality and healthiness were stronger considerations directing their food purchases. In contrast, consistent with prior research, low-SES parents more often reported cost as the guiding factor in their food purchases (Wright et al. 2015; Daniel 2016).

In addition to cost concerns, parents vocalized another important consideration: adolescents' own preferences. Parents across the socioeconomic spectrum reported that they faced pressures from their adolescents to buy certain foods, many of which they viewed as unhealthy. Parents reported differentially navigating these pressures. 96 percent of high-SES families had a parent, typically a mother, report that they regularly denied their adolescents' food requests for health reasons, compared to 54 percent of middle-SES families and 13 percent of low-SES families. ${ }^{6}$ This is not to say that low-SES parents did not ever deny their adolescents' requests; however, when they did, their reasons were usually financial. Among low-SES parents, 52 percent said that they only denied their adolescents' food requests if they lacked the money. When funds were available, low-SES parents reported buying the food their adolescents requested. Many low-SES parents also explained that, even when funds were tight, they strove to meet their adolescents' requests: 35 percent of low-SES parents said that they always bought what their adolescents asked for, even when those requests guided them away from cheaper, healthier food options.

That high-SES parents consistently denied adolescents foods they could easily afford whereas low-SES parents stretched their dollars to meet these requests highlights the varying meanings that parents attach to food across the socioeconomic spectrum. In the following three sections, I show how parents' differential navigation of adolescents' requests is reflective of food's symbolic value across the socioeconomic spectrum. To do so, I present three families-one high SES, one middle SES, and one low SES - from this study's observational phase. With each case, I draw heavily from interviews with parents to demonstrate the prevalence of each approach within their socioeconomic group.

\section{Low-SES Parenting and Food Provisioning}

Nyah Baker was a low-SES black single mother of two adolescent daughters, Mariah and Natasha. Nyah, who did not graduate from high school, provided for her family over the years by working various minimum-wage jobs. Similar to most low-SES families in this study, Nyah's financial situation was precarious. When I first met Nyah, she had most recently been employed cleaning college dorms until health troubles prompted her doctor to recommend a year's leave from work. Nyah was supposed to receive monthly disability payments, but four months into unemployment, she had yet to see any deposits into her bank account.

At the time of observations, Nyah's monthly income consisted of approximately \$190 in food stamps and \$200 in social security payments for her daughters' learning disabilities. To make ends meet, Nyah relied on the little money she had saved while working and the income that her boyfriend brought in through his informal 
employment as a neighborhood mechanic. Although Nyah could usually meet her subsidized rent, she was sometimes unable to consistently pay all her bills. During the time I spent with the Bakers, I observed the cancellation of both Nyah's cell phone plan and her cable service due to outstanding payments.

These tenuous financial circumstances shaped Nyah's daily life, including her approach to food provisioning. Like other low-SES parents in this study, Nyah described aspects of her family's diet as "unhealthy," explaining as follows:

I think we're eating way unhealthy because of a lot of the snacks. Of course I know that's junk food. I'ma keep it real. I buy a lot of junk food. Snacks like donuts, Nutty Buddies, the popcorn and stuff like that: chips, sodas. We always buy Hot Pockets, corn dogs. . .

Yet Nyah also believed that diet was vitally important for her and her adolescents' health. She told me, "I know we need to start eating healthy. What looks good and tastes good is not always good for you. I know that. I know that." Nyah frequently commented on the importance of a healthy diet, including one afternoon, when Nyah, Mariah, and I drove to the pawn shop to exchange Nyah's jewelry for cash. As Mariah sat in the front seat eating Fruity Pebbles out of the box, Nyah commented: "if you eat well, you're going to live long, that's for sure." Yet amidst concerted efforts to stay afloat, the healthiness of her daughters' diets did not always feel like the most pressing concern to Nyah.

What was often more salient to Nyah was the awareness that she had very limited means through which to give Mariah and Natasha what they wanted. Nowhere was this limitation clearer than when Natasha and Mariah were on summer break. Nyah's financial circumstances during this period challenged her abilities to keep her daughters safe and happy when funds to take the girls on vacation or enroll them in activities were scarce. Nyah did her best. One evening at summer's outset, she explained to her daughters that despite all that they lacked, there was still a great deal to be thankful for:

I told them it's not all about fancy clothes and fancy cars. Be happy you have a roof over your head, you have food in here, you can watch TV when you want to and use the bathroom whenever you want to. You have a fan inside to go to when you get hot and a blanket to get under when you're cold. . .

As Nyah recounted this story to me, she paused momentarily before concluding: "I'm just trying to survive."

A key component of Nyah's survival involved consistently denying her adolescents' requests for indulgences. Within this context of ongoing refusals of larger purchases and investments-such as enrollment in sports camps and arts programsNyah found that food offered a chance to say "yes" to her adolescents. Thus, Nyah and other low-SES parents aspired to grant their adolescents' food requests often. On a trip to the grocery store, when Natasha asked Nyah for a bag of Doritos for \$0.99, Nyah could say yes. When Mariah requested a Dr. Pepper for \$1.50, Nyah could oblige. In the afternoon, when the ice cream truck rolled around, Nyah routinely gave her daughters $\$ 2$ to spend on treats. These actions occurred alongside 
Nyah's frequent remarks to me that she should encourage her daughters to make other dietary choices. However, food offered Nyah an accessible and relatively inexpensive way to satisfy her daughters wants and get through each day. "It makes them happy," Nyah explained.

Low-SES parents' honoring of their adolescents' dietary requests not only served to compensate for broader material deprivation; meeting these requests also helped parents to derive a sense of worth as competent caregivers. So important were these priorities that low-SES parents explained that meeting their adolescents' requests could lead these parents away from the most affordable food choices. Faye Bautista, a low-SES Filipina mother, recounted that she spent around \$35 on take-out and delivery every day because it is what her 16-year-old daughter Melanie preferred. "We spend a lot of money for food," Faye lamented. "Whatever Melanie wants." The prioritization of adolescents' preferences was similarly on display one afternoon when I accompanied Nyah and Mariah on a trip to Starbucks. After paying the $\$ 10.80$ tab for their frappucinos, Nyah spent $\$ 25$ getting their eyebrows waxed at a nearby salon. With the financial hardship Nyah faced, that money could certainly have gone elsewhere: toward turning the cable back on or addressing longstanding household repairs. But Nyah put these expenses on the backburner in order to provide for her daughter's wants.

Many low-SES parents spoke about spending their last pennies to take their adolescents out to their favorite eating establishments, highlighting that they wanted their adolescents to feel that their preferences were heard and mattered. Miranda Kirk, a low-SES white mother who struggled to pay rent most months, noted she would still do anything to give her adolescent daughters the food they want:

The way I feel about food is, like, if they need food and they're hungry, then I'm gonna buy it. I don't care how much money is in my pocket. I'll spend my last \$20. That's how it is. It'll come. Sometimes it's hard. Everything, literally every dime I get goes to food. Every dime. But what do you do? I just buy it for them. It's for my babies. I love them more than anything on the planet. I don't care. They want it. They'll get it. One day, they'll know. They know I love them and that's all that matters. So what? It's food. I don't care. If she wants a \$2 candy bar, I get it for her if I have it.

For Miranda, food not only offered an important means of showing love and care to her daughters; she also saw her ability to get her daughters the food they wanted as important evidence that she was a responsible provider. Similarly, Delfina Carrillo, a low-SES Hispanic single mother who worked as a supermarket cashier, explained that her son's dinner typically came from whichever fast food restaurant he selected on a given night. Although Delfina was aware that she could save money by cooking dinner, her priority was her son's happiness:

"As long as my son eats, I'm happy. I always make sure that there's something that he likes so he'll eat. I guess the most important thing is something he likes so he will eat."

Being able to meet these requests allowed Delfina and other low-SES parents to affirm their adolescents and give them a sense of dignity in a context where daily 
life challenged that ability. Delfina, like other low-SES parents, even noted that sometimes she would eat something inexpensive or smaller for dinner, such as a can of soup, so that she could provide her son with food that he wanted.

Interview after interview with low-SES parents highlighted how, in a context of scarcity, responsible parenting meant honoring adolescents' preferences. Some mothers gave accounts like Elissa Garcia, a low-SES Hispanic single mother who did not take for granted the ability to oblige her adolescents' food wishes. Elissa recalled an earlier time when she found herself in even direr financial situation. "I had barely enough to pay the bills and not much to pay for food," she recounted. Those times required Elissa to say no to even the most inexpensive of her adolescents' food requests. Although at the time of the interview, Elissa still lived below the poverty line, she generally had enough money to offer her adolescents foods that they enjoyed. When there were extra funds-when Elissa worked overtime or encountered a supermarket sale-her first priority was getting her adolescents what they wanted:

They'll be like, 'Can I get some Hot Pockets, Mom?' Or 'Can I get some pizza bites?' [ . . . Or 'Can you get me a soda?' I think they pretty much have all the say they want [. . . I I want them to feel comfortable with what they're eating.

Although Elissa also explained that she did not want her adolescents eating those foods regularly, that consideration was outweighed by a much more pressing consideration. For Elissa, being a good parent meant being able to give adolescents not only enough but also some of what they wanted.

Finally, food's symbolic value was evidenced in how Nyah and Dana, the other low-SES mother I observed, spent the $\$ 300$ they received for participating in this study's observations: both mothers divided the payment equally between them and their adolescents. Nyah gave each of her two daughters $\$ 100$, all of which they spent within two weeks on candy, fast food, and clothes. Nyah used $\$ 60$ to pay the electricity bill and $\$ 20$ to purchase gas. With the remaining $\$ 20$, she bought beer and chips. Dana spent her money similarly, giving her two daughters each $\$ 100$ and spending her $\$ 100$ on a haircut. For Nyah and Dana, a windfall of cash did not result in an increase in fruit and vegetable consumption, as some scholars would suggest. Rather, this payment offered these low-SES mothers an opportunity to give adolescents what they wanted in a context when such opportunities were relatively scarce (Pugh 2004).

Although low-SES parents in this study valued their adolescents' diets and health, their food provisioning approaches were shaped in part by the meaning that food held in a context of severe financial constraints. Amidst limited chances to meet their adolescents' larger desires, accommodating their adolescents' relatively smaller wishes (for instance, for food) was usually still attainable. The situation was different for high-SES parents. Next, I show how these parents' greater resources altered food's symbolic value and their navigation of adolescents' requests. 


\section{High-SES Parenting and Food Provisioning}

Just twenty minutes up the road from the Bakers, in an upper-middle class neighborhood, lived the Cains, a high-SES white family of four. While her husband Mark worked as a lawyer, Heather had been a stay-at-home mom for the past fifteen years. That the Cains owned their two-story home suggested a degree of financial security, which Heather and Mark both confirmed. Although Mark wished that Heather was more cost-conscious in her food purchases, Heather noted that they were not worried about money. "I do try to stay within a reasonable amount, but I don't have a dollar amount set up," she explained.

Just like the low-SES parents in this study, paramount to Heather was being a responsible provider to her two adolescents, Jane and Evan. But the environment within which Heather and other high-SES parents raised their adolescents differed dramatically from Nyah's. Of the parenting challenges that Heather faced, worrying that her adolescents were going without was not one of them. While Jane was enrolled in dance classes, sports camps, and volunteer organizations, Evan spent his time performing in his private school's theater group. One afternoon, after dropping Jane off at volleyball camp, Heather and I drove the hour home to start preparing dinner. Heather told me during the car ride, "Jane has basically always gotten everything she wants."

According to Heather, one thing that both Jane and Evan consistently wanted was "junk food." Although her adolescents enjoyed fruits and vegetables, less healthy foods were their favorite. Heather explained that whenever she went grocery shopping, Jane and Evan had one request. "They always want junk. I mean, they really ask for a lot of it," she told me. However, how Heather navigated her adolescents' food preferences differed from the approach of Nyah and many other low-SES parents. Heather explained, proudly, how she responded to her adolescents' requests: "I just am pretty much like, 'No, no, and no."'

Similar to Nyah, Heather cared about the food that her adolescents consumed and derived a sense of worth as a caregiver from her food provisioning. However, Heather's socioeconomic position altered the symbolic meanings she attached to food. Absent concerns about providing enough, Heather derived feelings of provider worth through teaching Jane and Evan how and when to eat the "right" foods for the "right" reasons. Food was less so a compensatory medium and moreso a means through which to instill in her adolescents classed values about restraint and delayed gratification. Heather echoed many high-SES parents in explaining how she taught Jane and Evan about food. She told them the following:

We don't eat for comfort. We eat basically to nourish ourselves and to stay alive. Quantity-you've got to watch how much you put in your body. I want them to love food for many different reasons [. . .] Food is comfort when you're with your family. You think about Thanksgiving and these wonderful meals. But I also want them to think about, 'Well, I don't necessarily need all this food either. My body can do without it.'

Indeed, many high-SES parents explained that it was important to them that their adolescents developed values of self-restraint and control around food. Part and parcel of instilling these values was saying "no" to adolescents' requests for 
junk food. Through denying these requests, high-SES parents taught adolescents not only virtues such as self-control; parents simultaneously cultivated adolescents' palettes for foods that fit into their widely shared definitions of "healthy."

Certainly, Heather tired at times of hearing Jane and Evan complain about not getting enough of the foods they asked for. Yet her reluctance to honor these requests related to how she evaluated her success as a caregiver. Heather laughed one afternoon as she recounted to me the fights that she got in with Jane and Evan: “I don't buy enough junk food. They say, 'I don't ever have things in my lunchbox. You don't buy enough treats."' Yet Heather interpreted these complaints as evidence of responsible parenting. Whereas for Nyah, denying her adolescents the foods they wanted would have put their well-being at risk, the opposite rang true in many high-SES parents' minds: succumbing to adolescents' desires would jeopardize their health and their development (Cairns et al. 2013).

It is not the case, however, that adolescents' preferences were of no concern to high-SES parents. These parents reported going to great lengths to ensure that their adolescents' favorite healthier options were readily available and that their preferred dinners graced the table regularly. These parents also negotiated constantly with adolescents to find "better" substitutes for the foods they requested. For instance, when her daughters first asked for Cheez-Its, Patricia Adams, a highSES single white mother, was horrified. As a solution, Patricia set to work finding an alternative snack that she felt was acceptable, if not ideal:

I found a substitute at Whole Foods that had natural coloring, organic cheddar, no hydrogenated oil. So I buy things that I wouldn't normally buy but satisfy my teenagers.

Other parents employed similar tactics, using such negotiations as a tool to continue to teach their adolescents about self-control and restraint around food while simultaneously facilitating the cultivation of their palettes for certain foods over others.

That high-SES parents reported these negotiations as time-consuming and exhausting speaks to the strength of food's symbolic value within this group. It also underscores the abundance of resources that parents could draw on to partake in these negotiations. I witnessed the time and money that went into such food-related negotiations while watching Heather's efforts to provide novel, healthy foods that her adolescents would appreciate. One week, Heather visited the supermarket four times to ensure that the fridge was stocked with red and green grapes, Jane's preferred healthy snack. Another evening, I spent an hour helping Heather prepare a meal accompaniment that Jane had indicated interest in: a "tornado potato" dish made from spiral slicing, skewering, and roasting potatoes in a homemade sauce. As exhausting as these ongoing efforts were, Heather saw Jane's consumption of grapes rather than cookies and her enjoyment of homemade meals over fast food as evidence of a successful caregiving project. Thus, for high-SES parents, being a responsible parent meant cultivating in adolescents a specific consumption-related habitus (Wright et al. 2015).

If low-SES, single, working parents represented one end of the spectrum, parents like Heather represented the other: high-SES, married, stay-at-home. Although 
Heather's employment status certainly facilitated food provisioning, food's meaning to her was emblematic of its meaning to most high-SES parents, regardless of their employment status or family composition. Although high-SES parents who worked were certainly more pressed for time than Heather, they often drew on their financial resources to compensate for temporal constraints. Some families, like the Kapoors, a high-SES South Asian family, outsourced some of the labor associated with preparing food. Rohisha Kapoor, who worked full-time, explained that she had hired someone to come on the weekends to help cook meals for the week ahead. Similarly, Patricia, the single working mother mentioned earlier, noted that, instead of shopping around for the best deals, she would save time by buying more expensive products at Whole Foods:

I'm really busy and don't have a lot of time. In my ideal world, I would shop at Trader Joe's to get staples. Their bread is cheaper, and they've got a good selection. But I don't always have time to go there so then my backup plan is Whole Foods. I buy my produce at Whole Foods so then, if I'm limited on time, I end up getting a lot of my staples there too.

Thus, although high-SES parents varied in how much time they could allocate to food provisioning, their financial circumstances buffered against needing to consistently use food in a compensatory manner. In particular, busy high-SES parents' abilities to draw on available funds in food provisioning enabled them to continue to attach similar meanings to food as their stay-at-home counterparts.

Where variation did emerge among high-SES parents was between mothers and fathers' food approaches, highlighting the ways in which food's symbolic value is gendered. Although high-SES fathers broadly agreed with mothers about the importance of healthy eating, fewer said "no" to their adolescents' food requests. This finding was consistent with literature highlighting how food work and its relationship to cultivating children's health are central to motherhood (Beagan et al. 2017) but more peripheral to fatherhood (Fielding-Singh 2017). Notable, however, is that the two high-SES fathers in this study who were responsible for food largely resembled mothers both in the meanings they attached to food and how they navigated their adolescents' requests.

It is important to note that even though high-SES parents typically denied their adolescents' food requests, many high-SES adolescents reported still consuming these foods. Like middle- and low-SES adolescents, high-SES adolescents' exposure to foods and drinks outside the home- on television and through peers-helped cultivate in them a desire for products their parents disapproved of. High-SES parents, in their discussion of their adolescents' less healthy eating practices, offered additional insight into food's symbolic meaning to them. Many high-SES parents reported a sense of failure when their adolescents did not, in their eating practices, embody the values that these parents sought to transmit through food.

Amber Dawson, a high-SES white stay-at-home mother, was one of many who articulated such feelings. Amber approached her adolescents' diets similarly to Heather, but she struggled with her daughter Leann's pickiness. How Amber navigated this pickiness often involved giving in to Leann's unhealthy desires to ensure 
that Leann ate something. Amber felt highly conflicted about such submissions: "We're probably on the not-great end of the spectrum. I know kids who eat a lot better than my kids do." After explaining to me how Leann would systematically refuse fruits and vegetables but would happily drink Starbucks, Amber requested that I use a pseudonym to mask her identity. Just as high-SES parents took pride in their adolescents' healthy eating habits, many expressed guilt regarding moments when they felt they had failed to instill in their adolescents the "right" food practices.

\section{Middle-SES Parenting and Food Provisioning}

Qualitative studies of inequality often treat high- and middle-SES families as one socioeconomic group, referring to them as "middle class." I found that the middleSES families in my study were distinct from their high- and low-SES counterparts and that an examination of middle-SES families further elucidates how material conditions shaped food's symbolic value.

Middle-SES parents in this study shared commonalities with both their highand low-SES counterparts but were visibly trapped between competing ideals of responsible parenting. Although middle-SES parents resembled high-SES parents in their aspirations to transmit values around taste, health, and self-control, these parents' fewer resources precluded them from fully achieving these ideals. Middle-SES parents were forced to make trade-offs between these ideals and the practicalities of their everyday lives. This reality led middle-SES parents to occasionally use food as a compensatory tool to make up for constrained resources, which ranged from more limited financial means to longer working hours.

The Ortegas, a middle-SES Hispanic family, illustrated these trade-offs. Renata and her husband Miguel both worked full-time jobs: Renata as a bank manager and Miguel as a musician. Their two adolescents, Marcel and Daniela, resembled Heathers' adolescents in their extracurricular involvements. I spent many hours accompanying the Ortegas to activities such as church services, doctors' appointments, and sports practices. Renata, who was largely responsible for food provisioning, explained that their hectic schedules guided their consumption. Renata preferred to cook meals that could be on the dinner table in less than 20 minutes. Premade meals that could be popped in the oven last minute were a staple in the Ortegas' refrigerator and freezer.

Renata and other middle-SES parents' discussions of food bore a resemblance to those of high-SES parents, particularly in how they described their dietary goals for their adolescents and the constancy of adolescents' requests for "junk food." As Nan Braverman, a white middle-SES mother recounted about her two adolescent daughters: "it's dangerous to take them to the store with us because they're like, 'Ooh, can we have this? Can we have this? Can we have this?' [. . .] Chips or ice cream or those Arizona tea things." Although middle-SES parents like Nan aspired to deny such food requests, their material conditions shifted food's symbolic value and contributed to their navigation of these requests. Although some middle-SES parents said that they regularly denied their adolescents' requests, this was much less common than among high-SES parents. Nan's husband, Tim, captured a widely 
expressed sentiment expressed by middle-SES parents in pointing out, "It's the pick-your-battles thing, you know?" For middle-SES parents, saying "no" was less of the given that it was for high-SES parents.

Middle-SES parents also mirrored high-SES parents in their beliefs that they that they should be preparing homemade, healthy meals and limiting their adolescents' consumption of less healthy foods. Yet particularly for parents who worked long hours or were the sole breadwinners, food's symbolic value shifted from that of high-SES toward that of low-SES parents. Whereas high-SES parents reported occasionally having to make unwanted compromises related to food, middle-SES parents reported these compromises as more frequent and even commonplace. Whereas high-SES parents were often able to draw on their abundant financial resources to compensate for temporal constraints, middle-SES parents did not have these economic reserves to regularly uilize to such ends.

For instance, Renata explained that she did not feel that she always had the time or money to ensure that Marcel and Daniela were eating the way that she wanted. One compromise that Renata made revolved around her adolescents' preference for macaroni and cheese. Renata did not want to outright refuse this request because it was an easy dinner for her to put together and one that she knew Marcel and Daniela liked. Therefore, as a compromise, Renata chose to purchase stove-top brands over microwaveable options. She explained to me her rationale: "I try not to buy items that you cook in the microwave because those aren't really the best. So I try to make it something you can really quickly cook on the stove."

As much as middle-SES parents attributed these compromises to financial or temporal constraints, such compromises also represented a deep source of guilt for middle-SES parents. Catalina Ramos-Hayashi, for instance, was a middle-SES Hispanic married working mother who aspired to provision healthy foods to her daughter, which she defined as organic and homemade. She explained, "I feel like I'm being a better parent when I'm cooking and providing homecooked meals." Yet Catalina's daily two-hour commute meant that time to cook was relatively scarce in the evenings. These temporal constraints were accompanied by financial limitations that precluded her from purchasing high-quality products that would make routinely cooking easier, such as prechopped vegetables. Together, these limitations meant that Catalina all too often found herself resorting to what she saw as unhealthy take-out options. Like other middle-SES mothers, Catalina felt that she was acting as a less responsible parent when such compromises had to be made:

Whenever I'm having to buy food [out] every day I'm like, this is horrible! I'm pumping my family and myself full of junk. But sometimes I'm like, that's just the way life goes and it's ok.

Although Catalina's notions of responsible parenting around food mirrored Heather's, Catalina's context made it more difficult for her consistently accomplish these goals. Other middle-SES parents echoed Catalina's feelings about not always being able to provision the food they believed was best for their adolescents. Renata, for instance, repeatedly expressed guilt and embarrassment about the fact that delivery from Papa John's and Panda Express were weekly occurrences in her home, as this reality belied Renata's ideals for her adolescents' diets. Renata's desire 
to better meet these ideals, but the limitations of doing so, came to light one evening when Renata told me about a dream she had begun to entertain related to time constraints. I recorded this depiction in my field notes:

Renata tells me that her dream would be to hire someone who would get home a bit before her and start tidying up and cook them dinner. "That would be the best," she says. Renata says that she doesn't like to cook. She does it because she has to. She says that some people find it therapeutic and love to try new recipes, but that she is not one of those people. She has a friend who has a caretaker for her younger children, but that caretaker goes above and beyond and will also make dinner. Renata says that would be what she wants.

Renata felt that she was short-changing her adolescents by not consistently provisioning home-cooked meals, but unlike many low-SES parents, her anxieties did not center on providing enough. That, however, was not the case for all middleSES parents, particularly those whose financial circumstances placed them just above their low-SES counterparts. Food's symbolic value to these parents, like Chastity Banks, a black married mother, veered toward more compensatory. When Chastity's husband went back to school in early 2016, Chastity transitioned from staying at home to working full time. In my interview with Chastity months after this transition, Chastity expressed feeling guilty about having less time to devote to thinking about and shopping for food. Consequently, as part of her efforts to feel and signal that she was a responsible caregiver, Chastity ensured that she cooked her daughters whatever they asked for on the rare occasion when she could go into work late:

There are some mornings where I don't go to work early and I'll stay home and they're like, 'Oh my gosh.' I'll make a full breakfast. I'll make biscuit sandwiches like they have at McDonalds with the sausage. And they're like, 'Can you make this every morning?' I said, 'No. I don't have that kind of time. I'm just going in late today. So that's the only reason I'm here.'

Even though Chastity later explained that she did not believe the breakfast she prepared her daughters was "healthy," she nonetheless valued the opportunity to be there for her daughters. She expressed that it was meaningful for her to provide them with what they wanted, rather than what she felt she could normally scramble to put together for them. Like other middle-SES parents, Chastity's constraints shaped her use of food to signal to her adolescents that even though she was not around as much, she still cared deeply about them and their wants. In this way, food's symbolic value to middle-SES parents reflected a tension between fulfilling the consumption ideals of high-SES parents while compensating for perceived limitations in other domains. 


\section{Discussion and Conclusions}

This article highlights an important mechanism shaping parents' food provisioning across socioeconomic status: food's symbolic value. This symbolic value is forged in part through broader structural inequalities that configure the contexts within which parents raise and provide for adolescents.

I find that socioeconomic status shapes the meanings that parents attach to food, and those meanings help shape how parents approach their adolescents' diets. For low-SES parents, food is a symbolic antidote to a context of deprivation. Under the structural constraints and stresses of poverty, low-SES parents use food to compensate for other realms of scarcity. Doing so not only serves to emotionally satisfy adolescents, but it also bolsters parents' own sense of worth and competence as caregivers. In contrast, among high-SES families, food assumes a distinct but equally powerful symbolic function. For high-SES parents, food offers a medium for fulfilling different ideals of responsible parenting. These ideals involve fostering a particular set of values around self-restraint and health, all the while cultivating adolescents' palettes for acceptable foods. Using food this way also allows high-SES parents to feel and signal to others that they are successful, invested caregivers. For middle-SES parents, who parent in a precarious space of neither abundance nor scarcity, food's symbolic value vacillates between its meaning at the two ends of the socioeconomic pole. Middle-SES parents aspire to use food like high-SES parents in order effort to transmit similar values; however, these parents' more limited circumstances mean that they can also use food as a compensatory tool.

These findings are consistent with research showing that socioeconomic status underpins how parents navigate the dietary changes associated with adolescence (Eldridge and Murcott 2000; Kaufman and Karpati 2007). As adolescents become increasingly independent and subject to external influences that encourage augmented preferences for less healthy, calorie-dense foods, scholars have documented variation in the amount of responsibility for and level of control parents report exercising over their teenagers' food consumption. Low-SES parents largely exercise less control whereas middle-SES parents report more feelings of responsibility in continuing to shape teenagers' food practices (Backett-Milburn et al. 2010). This study's findings suggest that these different approaches may, in part, be mediated by food's symbolic value to parents. The low-SES parents in my study, although they do not view their adolescents' preferences as healthy, respect these preferences and largely seek to honor them rather than change them, even when doing so means more spending more. In contrast, many high-SES parents struggle to accept their adolescents' unhealthy preferences; their denial of these preferences and attempts to foster different preferences underscore the meanings they attach to the food their adolescents eat.

Relatedly, these findings importantly show that low-SES parents strive to meet their adolescents' food requests not because they subscribe to a "culture of poverty" (Lewis 1961) that devalues their adolescents' diets and health or because they are less knowledgeable or competent at food provisioning (Wright et al. 2015). Rather, I find that although parents across the socioeconomic spectrum value their adolescents' diets, their diverging material circumstances shape facets of their food provisioning 
approaches. Most mothers in this study subscribe to widespread discourses that frame their adolescents' diets and health as their responsibility (Brenton 2011; Kinser 2017) and attempt to meet these normative expectations. Low-SES mothers' provisioning strategies stem not from a disregard for their adolescents' diets or health; completely to the contrary, these parents' approaches reflect a deep desire to care and provide for adolescents amidst constrained material circumstances. This finding adds to mounting scholarship showing that differences in values do not fully explain the connection between socioeconomic status and parenting behaviors (Dohan 2003; Carter 2005; Edin and Kefalas 2005; Wilson 2009; Small, Hardin, and Lamont 2010; Lee and Zhou 2015).

That poverty not only deprives low-SES parents of financial resources but also shapes the meanings that become attached to food provisioning has important implications for understandings of dietary disparities. Whereas high-SES parents provision in an environment of abundance, security, and stability, low-SES parents do so in the face of scarcity, uncertainty, and instability (Sanchez-Jankowski 2008). Compared to high-SES parents' wealth of opportunities to provide for their adolescents and bestow unto them advantages like stable housing, schooling, and extracurricular activities, low-SES parents struggle to provide within severe resource constraints (Edin and Lein 1997; Chin and Phillips 2004; Tubbs et al. 2005; Pugh 2009; Beatty 2010; Desmond 2016). Although not the case within this study's sample, low-SES families in many regions live in neighborhoods that grant them limited access to affordable, healthy foods (Gordon et al. 2011) or that inundate parents and adolescents with cheaper, fast foods (Kwate et al. 2009). Beyond remedying spatial inequalities, policies aimed at durably bettering families' financial states may be most effective in reducing dietary disparities. Providing low-SES families with economic stability and security-at best elevating them out of poverty-would allow parents to meet more of their adolescents' needs and wants, thereby decreasing the symbolic value of food to compensate for deprivation in other domains.

This study has limitations. First, food's symbolic value to parents and parents' food provisioning strategies may differ geographically. Future research should explore the generalizability of this study's findings to other regions. Second, although this study focused primarily on parent-adolescent interactions, adolescents and parents are embedded in various environments, including neighborhoods, workplaces, and schools. To more fully understand food's symbolic meaning within families, scholars should examine how parent-adolescent interactions around food are shaped by these embeddings as well as by external influences such as the media and marketing from food and beverage industries. Finally, the cross-sectional nature of these data preclude me from assessing the causal impacts of food's symbolic value to parents and their food provisioning on adolescents' diets or dietary health. I hope that future research will assess the long-term implications of the processes this study identified on adolescents' dietary and diet-related health outcomes.

In addition to its scholarly contributions, this study implicates societal evaluations of parenting strategies across the socioeconomic spectrum. Adolescents' diets and diet-related health outcomes are often framed through the paradigms of failure and blame of the parents (Zivkovic et al. 2010; Brenton 2011). For the public, the image of a parent buying their child a soda invokes judgment and disapproval. 
Yet viewing parents as solely responsible for adolescents' diets incorrectly places the consequences of systemic inequalities on the backs of caregivers. In doing so, these paradigms obscure the myriad structural inequities that shape the conditions within which parents make daily choices. In order to address dietary disparities, scholars, policymakers, and practitioners alike must acknowledge and address the consequences of socioeconomic conditions for parents' provisioning strategies and adolescents' consumption.

\section{Notes}

1 In three high- and two middle-SES families, I was unable to obtain or closely approximate household income data. In these instances, I categorized families according to parents' levels of education.

2 When parents were of different ethnoracial backrounds, I categorized the family on the basis of the race and/or ethnicity of the primary caregiver or food provider. I use the term "Asian" to refer to South, Southeast, and East Asians.

3 All names of institutions, organizations, and individuals have been changed to protect anonymity.

4 In 67 of 74 families, I conducted an interview with at least one parent and at least one adolescent. When possible, I interviewed more than one parent and more than one adolescent. In 4 families, I conducted an interview with one parent (and no adolescents). In 3 families, I conducted an interview with one adolescent (and no parents).

5 To determine whether a family resided in a "food desert," I used the US Department of Agriculture's online Food Desert Locator. This allowed me to identify whether their home address fell within a neighborhood federally defined as a food desert.

6 These percentages refer to whether at least one parent in the family reported regularly denying their adolescents' food requests (i.e., in 95 percent of high-SES families, at least one parent indicated that they regularly denied requests). That I calculated these percentages using families as the unit of analysis is important because in seven high-SES families and five middle-SES families, I conducted interviews with two parents. Thus, calculating percentages by families rather than parents prevents an overweighting of families within which interviews with more than one parent were conducted.

\section{References}

Backett-Millburn, Kathleen, Wendy Wills, Mei-Li Roberts, and Julia Lawton. 2010. "Food and Family Practices: Teenagers, Eating and Domestic Life in Differing Socio-Economic Circumstances." Children's Geographies 8: 303-314. https ://doi .org/10.1080/14733285. 2010.494882

Beagan, Brenda, Gwen Chapman, Joseé Johnston, Debora McPhail, Elaine Power, and Helen Vallianatos. 2017. Acquired Tastes: Why Families Eat the Way They Do. University of British Columbia: UBC Press.

Beatty, Alexandra. 2010. Student Mobility: Exploring the Impacts of Frequent Moves on Achievement. Washington, DC: The National Academies Press.

Bourdieu, Pierre. 1984. Distinction: A Social Critique of the Judgment of Taste. Cambridge, MA: Harvard University Press. 
Bowen, Sarah, Sinikka Elliott, and Joslyn Brenton. 2014. "The Joy of Cooking?" Contexts 13(3): 20-25. https://doi.org/10.1177/1536504214545755

Brannen, Julia, and Ann Nilsen. 2005. "Individualisation, choice and structure: a discussion of current trends in sociological analysis." The Sociological Review 53: 412-428. https: //doi.org/10.1111/j.1467-954X.2005.00559.x

Brenton, Joslyn. 2011. Negotiating an Elusive Health Ideal: Mothers, Children, and the Contested Meanings of Health and Weight. Dissertation. North Carolina State University.

Cairns, Kate, Josée Johnston, and Norah MacKendrick. 2013. "Feeding the 'organic child': Mothering through ethical consumption." Journal of Consumer Culture 13(2): 96-117. https : //doi.org/10.1177/1469540513480162

Cairns, Kate, and Josée Johnston. 2015. "Choosing health: Embodied neoliberalism, postfeminism and the 'do-diet'." Theory and Society 44(2): 153-175. https ://doi .org/10 . 1007/ s11186-015-9242-y

Carter, Prudence. 2005. Keepin' it real: School success beyond black and white. New York: Oxford University.

Caspi, Caitlin E., Glorian Sorensen, S.V. Subramanian, and Ichiro Kawachi. 2012. "The local food environment and diet: a systematic review." Health Place 18(5): 1172-1187. https://doi.org/10.1016/j.healthplace.2012.05.006

Chapman, Gwen, and Brenda Beagan. 2003. "Women's perspectives on nutrition, health and breast cancer." J Nutr Educ Behavior 35(3): 135-141. https://doi .org/10.1016/ S1499-4046(06)60197-8

Charmaz, Kathy. 2006. Constructing Grounded Theory: A Practical Guide through Qualitative Analysis. Thousand Oaks, CA: SAGE Publications.

Chin, Tiffani, and Meredith Phillips. 2004. "Social reproduction and child-rearing practices: Social class, children's agency, and the summer activity gap." Sociology of Education 77(3): 185-210. https://doi.org/10.1177/003804070407700301

Cook, Daniel Thomas. 2009. "Semantic provisioning of children's food: Commerce, care and maternal practice." Childhood 16(3): 317-334. https://doi.org/10.1177/ 0907568209335313

Cooper, Marianne. 2014. Cut Adrift: Families in Insecure Times. Berkeley: University of California Press.

Croll, Jillian K., Dianne Neumark-Sztainer, and Mary Story. 2001. "Healthy Eating: What Does it Mean to Adolescents?" Journal of Nutrition Education and Behavior 33(4): 193-198. https://doi .org/10.1016/S1499-4046(06)60031-6

Daniel, Caitlin. 2016. "Economic constraints on taste formation and the true cost of healthy eating." Social Science and Medicine 148: 34-41. https ://doi .org/10.1016/j . socscimed. 2015.11 .025

Darmon, Nicole, and Adam Drewnowski. 2008. Does Social Class Predict Diet Quality? American Journal of Clinical Nutrition 87(5): 1107-1117.

Desmond, Matthew. 2016. Evicted: Poverty and Profit in the American City. New York: Crown.

Desoucey, Michaela. 2016. Contested Tastes: Foie Gras and the Politics of Food. Princeton, NJ: Princeton University Press. https://doi .org/10.1515/9781400882830

DeVault, Marjorie. 1991. Feeding the Family. Chicago: University of Chicago Press.

Dohan, Daniel. 2003. The price of poverty: Money, work, and culture in the Mexican American barrio. Berkeley: University of California Press.

Drewnowski, Adam, and Stephen E. Specter. 2004. "Poverty and obesity: the role of energy density and energy costs." American Journal of Clinical Nutrition 79: 6-16. 
Edin, Kathryn, and Laura Lein. 1997. Making Ends Meet: How Single Mothers Survive Welfare and Low-Wage Work. New York: Russell Sage Foundation.

Edin, Kathryn, and Maria Kefalas. 2005. Promises I can keep: Why poor women put motherhood before marriage. Berkeley: University of California Press.

Eldridge, Jane, and Anne Murcott. 2000. "Adolescents' Dietary Habits and Attitudes: Unpacking the Problem of (Parental) Influence." Health 4: 25-49. https : //doi . org/10. $1177 / 136345930000400102$

Evans, Alexandra, Karen Banks, Rose Jennings, Eileen Nehme, Cori Nemec, Shreela Sharma, Aliya Hussaini, and Amy Yaroch. 2015. "Increasing access to healthful foods: a qualitative study with residents of low-income communities." The International Journal of Behavioral Nutrition and Physical Activity 12(Suppl 1): S1-S5. https: //doi .org/10.1186/1479-5868-12-S1-S5

Fielding-Singh, Priya. 2017. "Dining with dad: Fathers' influences on family food practices." Appetite 117: 98-108. https://doi.org/10.1016/j.appet.2017.06.013

Gantz, Walter, Nancy Schwartz, James Angelini, and Victoria Rideout. 2007. "Food for thought: television food advertising to children in the U.S." Kaiser Family Foundation. Menlo Park CA: Henry J.

Gillies, Val. 2005. "Raising the 'Meritocracy': Parenting and the Individualization of Social Class." Sociology 39: 835-853. https ://doi.org/10.1177/0038038505058368

Gillies, Val. 2007. Marginalized Mothers: Empowering Working Class Experiences of Parenting. Routledge: Abingdon

Gordon, Cynthia, Marnie Purciel-Hill, Nirupa Ghai, Leslie Kaufman, Regina Graham, and Gretchen Van Wye. 2011. "Measuring food deserts in New York City's low-income neighborhoods." Health and Place 696-700. https://doi .org/10.1016/j . healthplace. 2010.12 .012

Graneheim, Ulla, and Berit Lundman. 2004. "Qualitative content analysis in nursing research: Concepts, procedures and measures to achieve trustworthiness." Nurse Education Today 24: 105-112. https://doi.org/10.1016/j.nedt.2003.10.001

Hanson, Margaret, and Edith Chen. 2007. "Socioeconomic Status and Health Behaviors in Adolescence: A Review of the Literature." European Journal of Behavioral Medicine 30: 263. https://doi.org/10.1007/s10865-007-9098-3

Hayes, Sharon. 1998. The Cultural Contradictions of Motherhood. New Haven: Yale.

Jiménez, Tomás. 2017. The Other Side of Assimilation: How Immigrants are Changing American Life. Oakland, CA: University of California Press.

Johnston, Josée, and Shyon Baumann. 2010. Foodies: Democracy and Distinction in the Gourmet Foodie Landscape. New York: Routledge.

Johnston, Josée, Michelle Szabo, and Alexandra Rodney. 2011. "Good food, good people: Understanding the cultural repertoire of ethical eating." Journal of Consumer Culture 11(3): 293-318. https://doi.org/10.1177/1469540511417996

Johnston, Josée, Alexandra Rodney, and Michelle Szabo. 2012. "Place, Ethics and Everyday Eating: A Tale of Two Neighborhoods." Sociology 46(6): 1091-1108. https : //doi .org/10. $1177 / 0038038511435060$

Kanjilal, Sanjat, Edward Gregg, Yiling Cheng, Ping Zhang, David Nelson, George Mensah, and Gloria Beckles. 2006. "Socioeconomic status and trends in disparities in 4 major risk factors for cardiovascular disease among US adults, 1971-2002." Arch. Intern. Med. 166 (21): 2348-2355. https://doi.org/10.1001/archinte.166.21.2348 
Kant, Ashima, and Barry Graubard. 2013. "Family income and education were related with 30- year time trends in dietary and meal behaviors of American children and adolescents." J Nutr 143(5): 690-700. https://doi .org/10.3945/jn.112.165258

Kaufman, Leslie, and Adam Karpati. 2007. "Understanding the sociocultural roots of childhood obesity: food practices among Latino families of Bushwick, Brooklyn." Social Science and Medicine 64(11): 2177-2188. https ://doi .org/10.1016/j . socscimed. 2007. 02.019

Kinser, Amber. 2017. "Fixing Food to Fix Families: Feeding Risk Discourse and the Family Meal." Women's Studies in Communication 40(1): 29-47. https://doi .org/10.1080/ 07491409.2016.1207001

Kwate, Naa Oyo, Chun-Yip Yau, Ji-Meng Loh, and Donya Williams. 2009. "Inequality in obesigenic environments: Fast food density in New York City." Health and Place 15: 364-373. https://doi.org/10.1016/j . healthplace.2008.07.003

Lareau, Annette. 2003. Unequal Childhoods. Berkeley: University of California Press.

Lee, Jennifer, and Min Zhou. 2015. The Asian American Achievement Paradox. New York: Russell Sage Foundation.

Lewis, Oscar. 1961. The Children of Sanchez. New York: Random House.

Leibowitz, Jon, J. Thomas Rosch, Edith Ramirez, Julie Brill, and Maureen Ohlhausen. 2012. "A review of food marketing to children and adolescents: follow-up report." Federal Trade Commission, Washington, DC.

Lindsay, Jo, and JaneMaree Maher. 2013. Consuming Families: Buying, Making Producing Family Life in the $21^{\text {st }}$ Century. New York: Routledge.

Lutfey, Karen, and Jeremy Freese. 2005. "Toward Some Fundamentals of Fundamental Causality: Socioeconomic Status and Health in the Routine Clinic Visit for Diabetes." American Journal of Sociology 110(5): 1326-1372. https : //doi .org/10 . 1086/428914

May, Vanessa. 2011. Sociology of Personal Life. Palgrave Macmillan: New York.

Miles, Steven, Dallas Cliff, and Vivien Burr. 1998. "'Fitting in and sticking out': consumption, consumer meanings and the construction of young people's identitiesJournal of Youth Studies 1(1): 81-96. https://doi.org/10.1080/13676261.1998.10592996

Phelan, Jo, Bruce Link, and Parisa Tehranifar. 2010. "Social Conditions as Fundamental Causes of Health Inequalities: Theory, Evidence and Policy Implications." Journal of Health and Social Behavior 51(1): S28-S40. https : //doi .org/10.1177/0022146510383498

Pugh, Allison. 2004. "Windfall Childrearing: Low-Income Care and Consumption." Journal of Consumer Culture 4(2): 229-249. https : //doi .org/10.1177/1469540504043683

Pugh, Allison. 2009. Longing and Belonging: Parents, Children and Consumer Culture. Berkeley: University of California Press.

Romagnoli, Amy, and Glenda Wall. 2012. "'I know I'm a good mom': Young, low-income mothers' experiences with risk perception, intensive parenting ideology and parenting education programs." Health, Risk E Society 14(3): 273-289. https : //doi .org/10.1080/ 13698575.2012 .662634

Ristovski-Slijepceniv, Svetlana, Gwen Chapman, and Brenda Beagan. 2008. "Engaging with healthy eating discourse(s): ways of knowing about food and health in three ethnocultural groups in Canada." Appetite 50(1): 167-178. https ://doi.org/10.1016/j .appet. 2007. 07.001

Sanchez-Jankowski, Martin. 2008. Cracks in the Pavement: Social Change and Resilience in Poor Neighborhoods. Berkeley: University of California Press. 
Sargent, Roger G., Shizuko Yagi, Hylan D. Shoob, Sara J. Corwin, Thomas Rogan, and John Wanzer Drane. 2002. "Differences in diet quality among fourth and seventh grade public school students in South Carolina." Journal of the South Carolina Medical Association 98(2): 54-60.

Small, Mario. 2009. "How many cases do I need?" Ethnography 10: 5-38. https : //doi .org/ $10.1177 / 1466138108099586$

Small, Mario, David Hardin, and Michèle Lamont. 2010. "Reconsidering Culture and Poverty." The ANNALS of the American Academy of Political and Social Science 629(1): 6-27. https://doi.org/10.1177/0002716210362077

Stead, Martine, Laura McDermott, Anne MacKintosh, and Ashley Adamson. 2011. "Why healthy eating is bad for young people's health: Identity, belonging and food."Social Science and Medicine 72: 1131-1139. https://doi .org/10.1016/j .socscimed.2010.12. 029

Tubbs, Carolyn, Kevin Roy, and Linda Burton. 2005. "Family ties: Constructing Family Time in Low-Income Families." Family Process 44: 77-91. https://doi .org/10.1111/j . $1545-5300.2005 .00043 . x$

Turrell, Gavin, Belinda Hewitt, Carla Patterson, Brian Oldenburg, and Patricia Gould. 2002. "Socioeconomic differences in food purchasing behaviour and suggested implications for diet-related health promotion." Journal of Human Nutrition and Dietetics 15: 355-364. https://doi.org/10.1046/j.1365-277X.2002.00384.x

Wang, Dong, Cindy Leung, Yanping Li, Eric Ding, Stephanie Chiuve, Frank Hu, and Walter Willett. 2014. "Trends in Dietary Quality Among Adults in the United States, 1999 Through 2010." JAMA Intern Med. 174(10): 1587-1595. https://doi.org/10.1001/ jamainternmed.2014.3422

Wilson, William Julius. 2009. More than just race: Being black and poor in the inner city. New York: Norton.

Wills, Wendy, Kathleen Backett-Milburn, Mei-Li Roberts, and Julia Lawton. 2011. "The Framing of Social Class Distinctions Through Family Food and Eating Practices." The Sociological Review 59: 725-740. https://doi .org/10.1111/j.1467-954X.2011.02035.x

Wright, Jan, JaneMaree Maher, and Clare Tanner. 2015. "Social class, anxieties, and mothers' foodwork." Sociology of Health and Illness 37(3): 422-436. https://doi.org/10.1111/ 1467-9566. 12202

Zivkovic, Tanya, Megan Warin, Michael Davies, and Vivienne Moore. 2010. "In the name of the child: the gendered politics of childhood obesity." Journal of Sociology 46(4): 375-92. https://doi.org/10.1177/1440783310384456

Acknowledgements: This research was supported by Stanford University's Vice Provost for Graduate Education and the Department of Sociology. I thank Tomás Jiménez, Michelle Jackson, Doug McAdam, Jeremy Freese, Christopher Gardner, Marianne Cooper, Caitlin Daniel, Kristine Kilanski, Aliya Rao, Melissa Abad, Jennifer Wang, Anshuman Sahoo, Adrienne Frech, and the students in my course, "The Social Determinants of Health," for their constructive feedback on various drafts of this article. I am grateful to my collaborators at Hillview Central High School as well as to the families who participated in this research and shared their insights and experiences.

Priya Fielding-Singh: Department of Sociology, Stanford University. E-mail: priyafs@stanford.edu. 